\title{
Simulation of Computation and Experimental Investigations about Water Hammer in High Lift Water Transmission Pipeline
}

\author{
Ma Jun ${ }^{1, a}$, Wu Jiande ${ }^{1,2, b}$, Wang Xiaodong ${ }^{1,2, b}$ \\ ${ }^{1}$ Faculty of Information Engineering and Automation, Kunming University of Science and \\ Technology, Yunnan, Kunming, 650500, China \\ ${ }^{2}$ Engineering Research Center for Mineral Pipeline Transportation YN, Kunming, 650500, China \\ ae-mail: majun_km@foxmail.com, be-mail:km_trans@foxmail.com
}

Keywords: High Lift; Pump-stopping Water Hammer; Rapid Butterfly Check Valve; Air Valve.

\begin{abstract}
For the study on water hammer protection problems of long distance, high lift and a pressurization hoisting for long distance water supply system, the paper analyzes a long distance water transmission system whose high lift is up to 2100 meters by a pressurization hoisting in southwest china. The water hammer protection model is established for high lift and long distance water transmission pipeline based on fluid mechanics, and the solution of decision model is attained by Bentley Hammer software. Then, a comparative analysis on the hydraulic characteristics is elaborated based the model solution whether the pipeline is under the prevention measure or not. The experimental results show that the damage of pump-stopping water hammer is more serious and the water column separation phenomenon probably happens without the prevention measure. When the pipeline is installed a comprehensive water hammer device with the two stage of rapid butterfly check valve and air valve, it can effectively prevent the accidental caused by pump-stopping reverse, the damage of pump-stopping water hammer and the water column separation, and ensure the safe and stable operation of the pipeline.
\end{abstract}

\section{Introduction}

With the rising of "South water to North Diversion Project", concentrate outwards transportation in the west complicated region and water transmission project of municipals at all levels, the research on pipeline hydraulic model and its application has attracted great attention of general scholars and enterprises. Furthermore, the water hammer protection of water pipeline which is located in the high lift, large gap and undulating terrain is the most extensive research topic and an important guarantee for the water pipeline operate safely and stably [1] [2].

In the theoretical field, many theories lay an important theoretical foundation for the study of water hammer, such as Jin Zhui's "pump-stopping water hammer and its protection" [3], Wang Shuren's "water hammer Theory and its calculation" [4] and the comprehensive report of "Hydraulic Transients With Water Column Separation" [5], especially, the solving method of water hammer model based on characteristic line. In the field of application, the commonly protective measures are installing the one-way pressure regulating tower, air valve, butterfly valve and steam valve in the pipeline based on the hydrodynamics and the boundary conditions [6-9].

However, it is most likely that the water hammer occurred in the long distance pressured pipeline, especially the pipeline in high lift, many ups and downs, and long distance with high pressure [2]. Therefore, the research on water hammer prevention of pipeline in high lift, many ups and downs, and long distance with high pressure has become one of the research hotspots. In order to control the water hammer pressure of long distance pressured water pipeline, Wang Wenquan et al. [14] has calculated the pumping accident process of a long distance pressure water pipeline based on the characteristic line method, and puts forward the relative optimal slow closed butterfly valve closing time and closing mode. Aiming at the ultra lift up to 370 meters and a pressurization hoisting of the long distance water transfer system, Liu Jianhua [1] has established water hammer protection model, and solved with the characteristic line method. Respectively simulate and analyze the water hammer protection effect of pipeline that installed with two stage control valve, one-way surge tank and pressure tank etc, and put forward the plan of rational prevention. 
Combining with a long distance water transmission system whose ultra lift up to 2100 meters and with single pressure lifting, a high lift water pipeline water hammer mathematic model which is based on fluid mechanics is established, and it is solved by Bentley Hammer. Then doa comparative analysis on the hydraulic characteristics whether the pipeline is under the prevention measure or not. When the pipeline is installed a comprehensive water hammer device with the two stage of rapid butterfly check valve and air valve, it can effectively prevent the accidental caused by pump-stopping reverse, the damage of pump-stopping water hammer and the water column separation phenomenon, and ensure the pipeline to operate safely and stably.

\section{Calculation Model and Boundary Conditions of Water Hammer}

The model of water hammer is established by combining the motion equation, continuity equation and pipeline boundary condition together in the fluid motion. The model used in this paper is expressed briefly and pecific statement. The method to this question can be seen in literature [4].

Calculation Model of Water Hammer. the theory of elastic water column, water hammer motion equation and continuity equation can be expressed as follows:

The motion equation:

$$
\frac{\partial H}{\partial x}+\frac{1}{g} \frac{\partial V}{\partial t}+\frac{V}{g} \frac{\partial V}{\partial x}+\frac{f}{D} \frac{V|V|}{2 g}=0
$$

The continuity equation:

$$
\frac{\partial H}{\partial t}+V\left(\frac{\partial H}{\partial x}+\sin \beta\right)+\frac{c^{2}}{g} \frac{\partial V}{\partial x}=0
$$

In the above equations: $H$ is the pipe head $(\mathrm{m}) ; x$ is position coordinates, the provisions of the positive direction is pointing to the valve; $g$ is acceleration of gravity; $V$ is the flow velocity of fluid in pipeline $(\mathrm{m} / \mathrm{s})$, the provisions of the positive direction is pointing to the valve. $t$ is time $(\mathrm{s})$; $f$ is the pipe friction coefficient; $D$ is the pipe diameter; $\beta$ is angle between pipe and horizontal; $c$ is the water hammer wave propagation velocity, using the equation (3) to calculate.

$$
c^{2}=\frac{K}{\rho}\left(1+\frac{K D}{E e}\right)^{-1}
$$

In the equation (3), $\rho$ is the fluid density, $E$ is pipe wall material elastic modulus; $e$ is the thickness of the pipe wall; $K$ is the bulk modulus of water.

The Boundary Condition of Butterfly Check Valve. Assuming that the flow through the valve is $Q$, head loss caused by the valve is $\Delta H$, the relationship is established by the equation (4) and (5):

$$
\begin{aligned}
& \Delta H=\zeta \frac{Q^{2}}{2 g A} \\
& Q=C \sqrt{2 g \Delta H}
\end{aligned}
$$

In the equation (4) and (5), $\zeta$ is the valve resistance coefficient, which can be calculated using the equation (6); $A$ is pipeline sectional area; $C$ is the valve open area multiplied by the coefficient of flow.

$$
\zeta=\frac{A^{2}}{C^{2}}
$$

Assumed that the resistance of positive flow is approximately zero for the ordinary swing check valve, the valve is instantaneously closed when reverse flow began in a very short period of time $\left(Q_{P}=0\right)$.

The Engineering Introduction for Water Hammer Protection. The pipeline is located in the southwest plateau area in China. It is the water supply and return water pipeline of iron concentrate transporting, which has important significance to ensure normal production and solve the problem of local water shortages. The elevation of pipeline starting point is 1187 meters. The elevation of terminal impounding reservoir is 3210 meters. The elevation difference is 2023 meters; the designed water flow is $0.0195 \mathrm{~m}^{3} / \mathrm{s}$; the designed length of pipeline is $10.7 \mathrm{~km}$ which is expressed by 
$L$. the pipeline diameter is $168.3 \mathrm{~mm}$ (external diameter) which is expressed by $D$. The pipe wall thickness is $9 \mathrm{~mm}$, which is expressed by $e$; the material is the API-5L-X60 bare steel; Pipeline engineering is pump single water, there is a pumping station at the head end, which equipped with two high pressure plunger pump(with a prepared). The designed head is 2040 meters; the pressure of pump outlet is 23MPa; the flow is about $0.033 \mathrm{~m}^{3} / \mathrm{h}(120 \mathrm{t} / \mathrm{h})$.

Due to the pipeline has the high dropping, the pump is equipped with fast check valve at its exit to prevent pump-stopping water hammer and the flow back reverse. At the same time, the pipeline is installed the air valve to eliminate the influence on water column separation. The design of the pipeline longitudinal plane is shown in Figure 1.

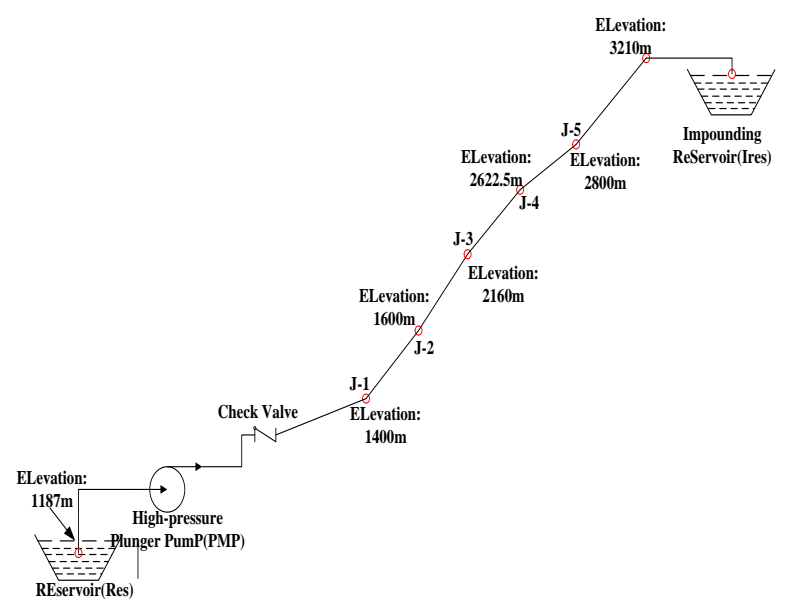

Fig.1. The design of the pipeline longitudinal plane

\section{Experiment Result Analysis}

The Pipeline Steady-state Results Analysis. The pipeline steady-state results attained by Bentley Hammer software are shown in Figure 2. The blue line is the hydraulic gradient in the normal conditions. The yellow line is the pipeline center elevation.

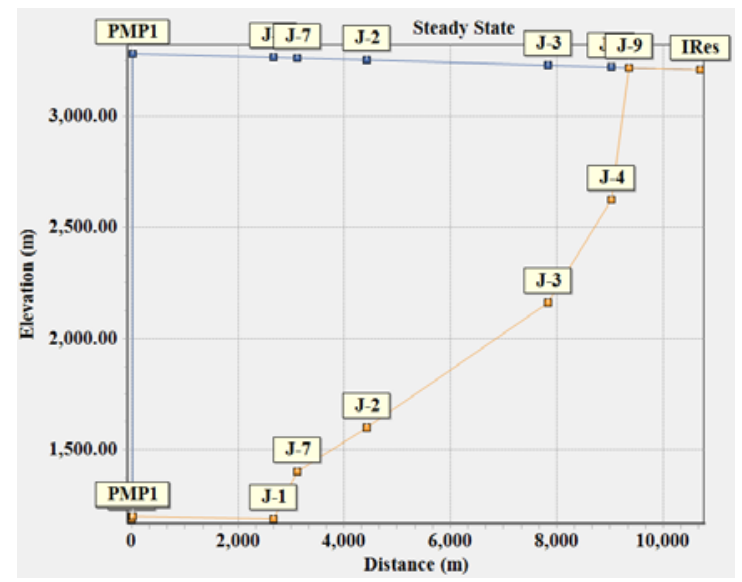

Fig.2. The pipeline center elevation and the water conservancy slope

The Calculation and Analysis of the Water Hammer without Prevention Measure. The pipeline sets nothing water hammer preventing device but the main pump, the main motor, the export service valve, the hydraulic control valve, the drainage system and the electric control system. When the pump accidental stops, the air content and the head envelope line of all computing nodes are shown in Figure 3. The red line in the graph represents the highest hydraulic envelope, the highest hydraulic envelope is represented by the blue line, the black line represents the work-time hydraulic envelope. It can be seen from Figure 3, the highest hydraulic envelope is far higher than the normal work envelope when pumping accidents happen, the safety coefficient is low, and there is a serious risk of the water hammer. At the same time the emergence of $0.045 \mathrm{~m}^{3}$ air appears in the $\mathrm{J} 7$ node, it may produce a water column separation. 


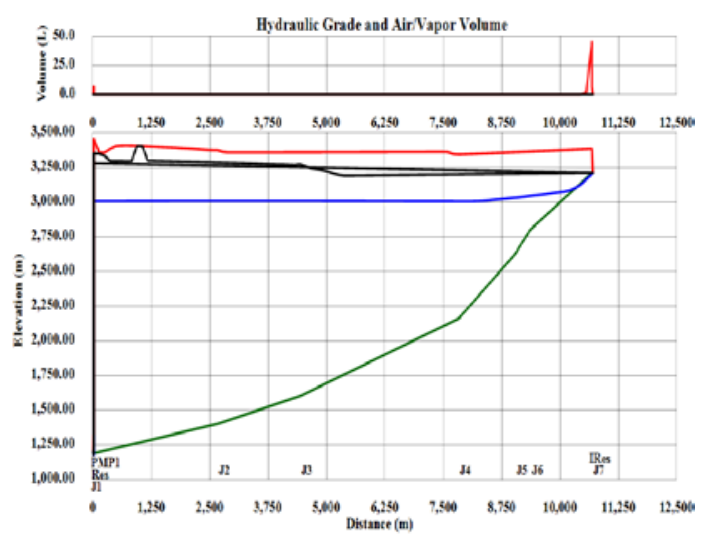

Fig.3. The air content and the head envelope line of all computing nodes

The Calculation and Analysis of Provention Measures for Water Hammer. In order to prevent the back flow of the water flow and the pressure pipeline pump reversal, it is necessary to set up the two stage pipeline control check valve. At the same time, water hammer can be prevented by controlling closing time and angle speed of the check valve. When the pump stops due to a fault occurs, the air content and head envelope of the pipeline all computing nodes are as shown in the Figure 4.

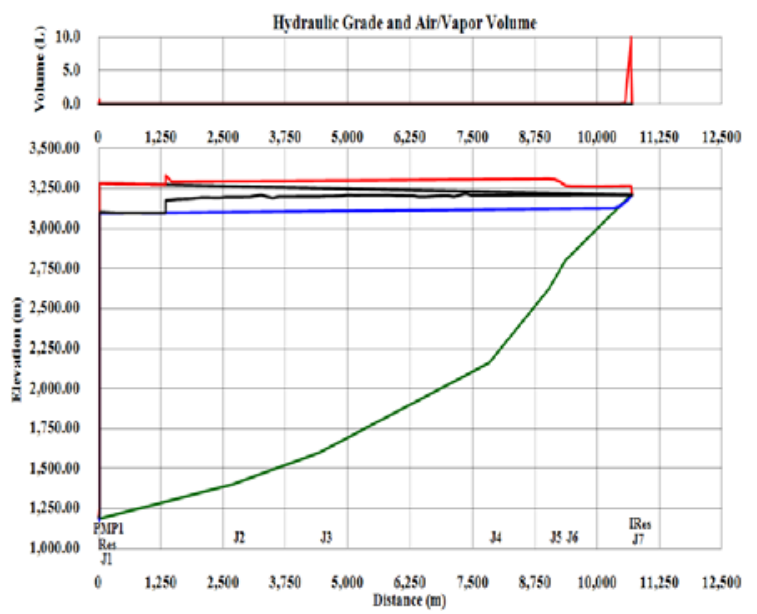

Fig.4. The air content and head envelope of the pipeline all computing nodes for the pump with the two stages of rapid butterfly check valve stopped due to a fault occurs

As it can be seen from Figure 4, the highest hydraulic envelope and the normal work of water conservancy slope is basic quite, and the risk of water hammer is controlled effectively. What's more, the air produced in $\mathrm{J} 7$ is only $0.001 \mathrm{~m}^{3}$, where the possibility of water column separation may be smaller.

However, the air still generates in the $\mathrm{J} 7$ node, and there is a risk of water column separation. Therefore, the air valve is setted in J7 node to eliminate the dangerous of water column separation. At the same time, the air content and head envelope of all computing nodes when the pump stops due to a fault occurs are as shown in the Figure 5. As it can be seen from Figure 5, when the pump in which the pipeline is setted two stage fast check valve and air valve stops, the water hammer impact and the water column separation resulted from pump stopping are in better control. 


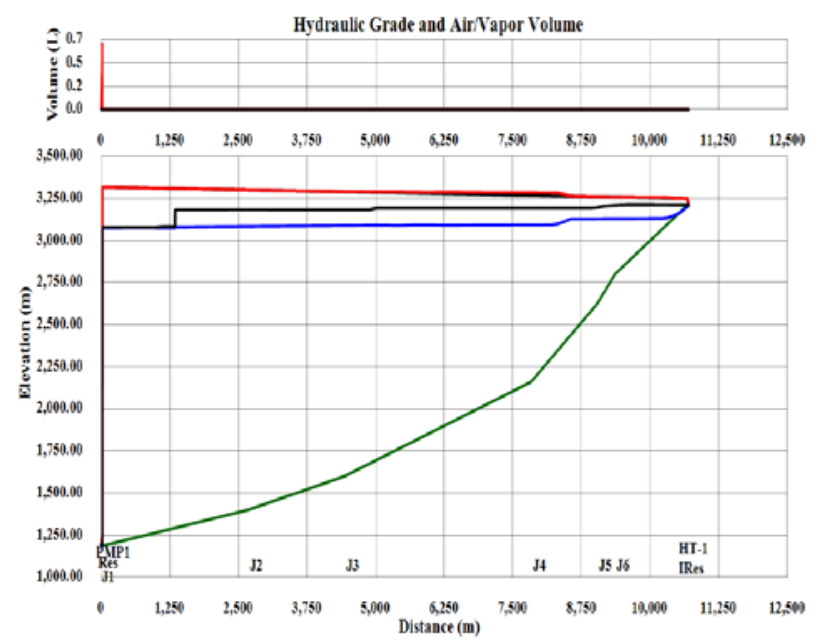

Fig.5. The air content and head envelope of all computing nodes for the pump with two stages of rapid butterfly check valve and air valve stopped due to a fault occurs

\section{Conclusions}

Aiming at the long distance water conveyance system of ultra high lift up to $2100 \mathrm{~m}$ and one time pressure lifting, a high lift water hammer mathematical model is established based on the principles of fluid mechanics in this paper which can be solved and analyzed by Bently Hammer software. From the experimental results, some useful conclusions can be made as follows:

(1)At the pump exit , the installation of two stage rapid butterfly check valve can effectively prevent stopping the pump backflow and suppress the water hammer effect, but it cannot completely eliminate the water column separation;

(2)Under the joint protection measures of installing two stage rapid butterfly check valve at the exit of the pump and laying air valve at the pipeline key nodes, it not only effectively prevent stopping the pump backflow and suppress the water hammer effect, but also completely eliminate the influence of column separation.

This paper establishes a water hammer protection model based on 2100 meters ultra high lift water pipeline, do an analysis comparison and put forward effective measures of water hammer protection. But this pipeline just simplify layout the processing of the actual engineering. In the actual engineering application, pipeline layout is needed to conduct a detailed calculation, at the same time, it carries a comprehensive analysis on the economic costs and other effects of water hammer protection to select water hammer protection scheme.

\section{Acknowledgements}

*Jiande Wu is the corresponding author. This work is supported by National Natural Science Foundation of China (No. 51169007), Science \& Research Program of Yunnan province (No.2011CI017 \& 2012CA022 \& 2013DH034).

\section{References}

[1] Liu Jianhua, Diao Meiling, Wang Chunyan, et al., Study on optimal surge protection for high-undulate long-distance water transmission pipeline. Journal of Harbin University of Commerce(Natural Sciences Edition) [J]. 2012 28(2) 183-186 193.

[2] Yang Yusi, Xu Yanyan, Xian Juzhi, et al., Research on water hammer prevention in high-life, hilly and long distance water transmission pipeline.Water \& Wastewater Engineering [J]. 2009 45(4) 108-111.

[3] JinZhui, et al., Pump-stopping water hammer and its protection. Beijing: China Architecture \& Building Press [M]. 2004. 
[4] Wang Shuren, Theory and its calculation of water hammer. Beijing: Tsinghua University Press [M]. 1981.

[5] Cabrera.E. and Fanelli.M.A., eds. Hydrndic transients with water column separation. Int. Assoc. for Hydraulic Research. Valencia. Spain [J]. 1991 159-181.

[6] Lu Suiju, Feng Minquan, Li Chunguang, Study on Numerical Simulation of water hammer pump water pipeline and its protection. Journal of Northwest A \& F University(Natural Science Edition) [J]. 2014 42(9) 219-226.

[7] Liu Zhuqing, Bi Huili, Wang Fujun, et al. Effect of air valves on water hammer protection in pressure pipelines.Journal of Drainage and Irrigation Machinery Engineering [J]. 201129 (4) 333-337.

[8] Yang Kailin, Air-valve surge tank for controlling liquid column separation in water supply projects. Journal of Hydraulic Engineering [J]. 2011 42(7) 805-811.

[9] Wang Wenquan, Zhang Lixiang, Yan Yan, Optimization of Closing Ways of Slow Closing Check Valve by Accidental Pump-Stop in Pressure Water Supply Pipeline.Transactions of Beijing Institute of Technology [J]. 2011 31(10) 1135-1138 1152. 